\title{
ANGER IN PROTEST NETWORKS ON TWITTER
}

\author{
Nazmiye Gizem Bacaksizlar ${ }^{1}$, Samira Shaikh ${ }^{2}$ and Mirsad Hadzikadic ${ }^{2}$ \\ ${ }^{1}$ Santa Fe Institute, Santa Fe, New Mexico, USA \\ ${ }^{2}$ University of North Carolina at Charlotte, Charlotte, North Carolina, USA
}

\begin{abstract}
We examine the influence relationships in online social networks and the distribution of anger during protests in Charlotte, North Carolina (2016) and Charlottesville, Virginia (2017). We extracted mention and retweet networks of users tweeting during these protests, and conducted emotion analysis on tweets to determine anger level of the users. We found that users with most followers receive most mentions and retweets. Anger levels peaked early on and then tapered off during both protests. The angriest users received few mentions and retweets. Our research has a potential to help understand influence dynamics in protest communication networks and provides insights for public policy implications.
\end{abstract}

\section{KEYWORDS}

Social Networks, Anger, Emotion, Twitter, Natural Language Processing, Protests

\section{INTRODUCTION}

Large political protests in the real-world are often reflected in numerous discussions on social media platforms. We investigate emotional contagion (specifically anger) in protest-related discussions on Twitter. The main research questions are: 1) What is the network structure of social media users who discuss protests on social media? 2) What is the level of anger among the users discussing the protests? 3) What is the relationship between the network position and anger level of the users?

Actual and potential protesters use Twitter and/or Facebook to connect with each other, communicate about the protests, and plan and organize their activities (Tufekci \& Wilson, 2012, Valenzuela, et al., 2014). Social science theories on a threshold for political protest participation are helpful to define network structures for users (Granovetter, 1978 , Xie, et al., 2011). It has also been found that Twitter has an influence on spreading information and emotion in social networks (Anger \& Kittl, 2011, Jansen, et al., 2009, Mitchell, et al., 2013, Taxidou \& Fischer, 2014). However, there is a dearth of studies on how mention/retweet network structure influences spread of emotions related to protests (Tremayne, 2014). Here we focus on the spread of anger. Past studies have shown that anger is the triggering emotion for protests (Castells, 2015), but it has not yet been studied how anger spreads on social media after the protests have started.

We investigate contagion of anger in tweets after two recent protests: One occurred in 2016 in Charlotte, NC, where the police shooting of Keith Lamont Scott, who was African American, triggered the Black Lives Matter protests. These protests are known as the Charlotte uprisings or Charlotte riots (Lee, et al., 2016). The other is the Unite the Right rally that occurred in Charlottesville, VA in 2017. This protest, also known as the Charlottesville rally or Charlottesville riots, was a white supremacist rally (Astor, et al., 2017).

\section{METHOD}

To answer our research questions, we used a combination of empirical analysis of Twitter networks and natural language processing algorithms to detect anger levels of each user in the corresponding social network. In the first step, we gained access through Twitter' s proprietary firehose (Gnip Historical PowerTrack) to tweets that were sent in response to the two protests mentioned above, which used specific hashtags and keywords during the relevant protest periods. For Charlotte protests, between September 20 - 26, 2016, we extracted 1.36 million tweets. To identify the relevant tweets, only tweets with 
at least one of seven protest-related hashtags and keywords (e.g., \#KeithLamontScott, \#CharlotteProtest) were included in the data analysis. For Charlottesville protests, we extracted 390,915 tweets with relevant hashtags and keywords (e.g, \#standwithcharlottesville, \#HeatherHeyer) between August 11 - 18, 2017. These data sets have 430,260 (Charlotte) and 188,516 (Charlottesville) unique users. We conducted a comparative analysis for Charlotte and Charlottesville' s protests with these two datasets.

\subsection{Network Analysis}

We extracted user networks for both protests using mentions and retweets. Mentions in tweets appear as "@username". With retweeting, users simply copy the posted tweet from the target user. We call the user who mentions or retweets another user the source user, and the user who is mentioned or whose tweet is retweeted by the source user the target user. The resulting graph is a weighted directed network with links from the source to the target user weighted by the number of mentions or retweets of the target user by the source user.

We use this graph to investigate the influence level distribution between source and target users. We divided the users in two categories: influential users (those who have more than 10,000 followers) and general public users (with less than 10,000 followers). The most active influential users in our dataset were media channels, politicians, journalists, celebrities, singers, comedians, and actor/actresses. We select only active (not suspended) users and their non-duplicated tweets. We also remove all self-mentions. The network graph visualizations were created with the Gephi software (Bastian, et al., 2009). To have the datasets from the two protests more comparable, we sampled 5,000 tweets from each dataset (Hu \& Lau, 2013), which were retweeted or mentioned by one or more source users.

\subsection{Emotion Analysis}

To detect anger, the Linguistic Inquiry and Word Count, or LIWC, analysis is applied on each tweet (Pennebaker, et al., 2015). LIWC is a text analysis application for language and disclosure studies, explorations with an expanded dictionary and a modern software designed in 2015. In the LIWC dictionary, words are categorized in different emotions and the frequency of words denoting each emotion are compared to overall number of words. We derived the level of anger for each tweet and calculated mean anger for each user over all of his or her tweets.

\section{RESULTS}

\subsection{Weighted Graphs with Directed Links}

For visualization purposes, the weighted graphs with directed links represent users as nodes, links between nodes as mentions or retweets of tweets, and node sizes as the number of mentions or retweets made for the node's tweets. A tweet can have more than one mentioned users. In the Charlotte's network, there were 6,259 nodes (all source and target users) and 5,656 edges. Figure 1(a) shows different communities in the network. The colors denote possible different communities in the network. The nodes with the highest in-degree tend to be influential users. For instance, users mentioned the Charlotte Mecklenburg Police Department, media channels (e.g., CNN), and one of the Black Lives Matter activists the most. The modularity score of this network structure is quite high: $97 \%$. A high modularity score indicates possible presence of community structure, which means dense connections between the nodes within modules/groups but sparse connections between nodes in different modules (Newman, 2006).

In the Charlottesville network, there were 5,981 nodes (all source and target users) and 5,719 edges. Figure 1(b) shows different communities in the network. Again, the colors denote possible different communities in the network. In the same fashion as the Charlotte Twitter data set, most of the mentioned users in Charlottesville are also influential. This shows that influential users, such as Donald Trump and social media platforms (e.g., Facebook and Instagram), occur as the highest in-degree nodes. This might indicate that Twitter users aimed at taking the government attention and sharing tweets about the protest on social media. The modularity score of this network is a bit lower than for Charlotte protest: $90 \%$. 

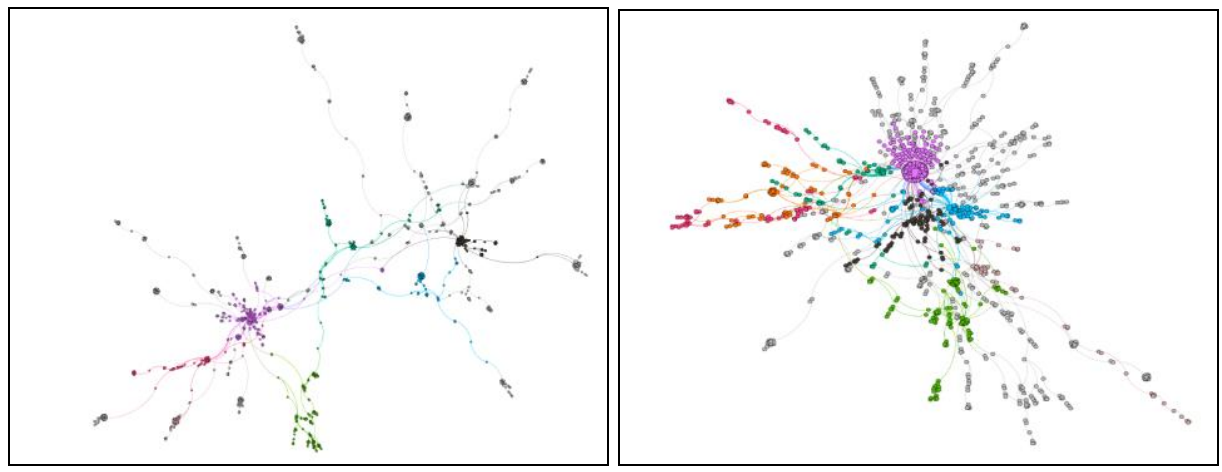

Figure 1. Network Sample for the (a) Charlotte Dataset Sample and (b) Charlottesville Dataset Sample

\subsection{Anger Distributions across Protest Datasets}

Figure 2(a) shows the mean anger per hour of the top ten angriest users (Max Anger), of the 32\% of tweets that showed at least some anger (Mean Non-zero Anger), and of all users (Mean Anger), for the Charlotte protests. During the second day of the Charlotte protests, the anger level peaked which was likely due to the announcement that the Charlotte-Mecklenburg Police Department chose not to release the video of the shooting of Keith Lamont Scott. The percentage of users showing some anger in the Charlotte dataset is $47 \%$. That means that angry users also tweet less than non-angry users.
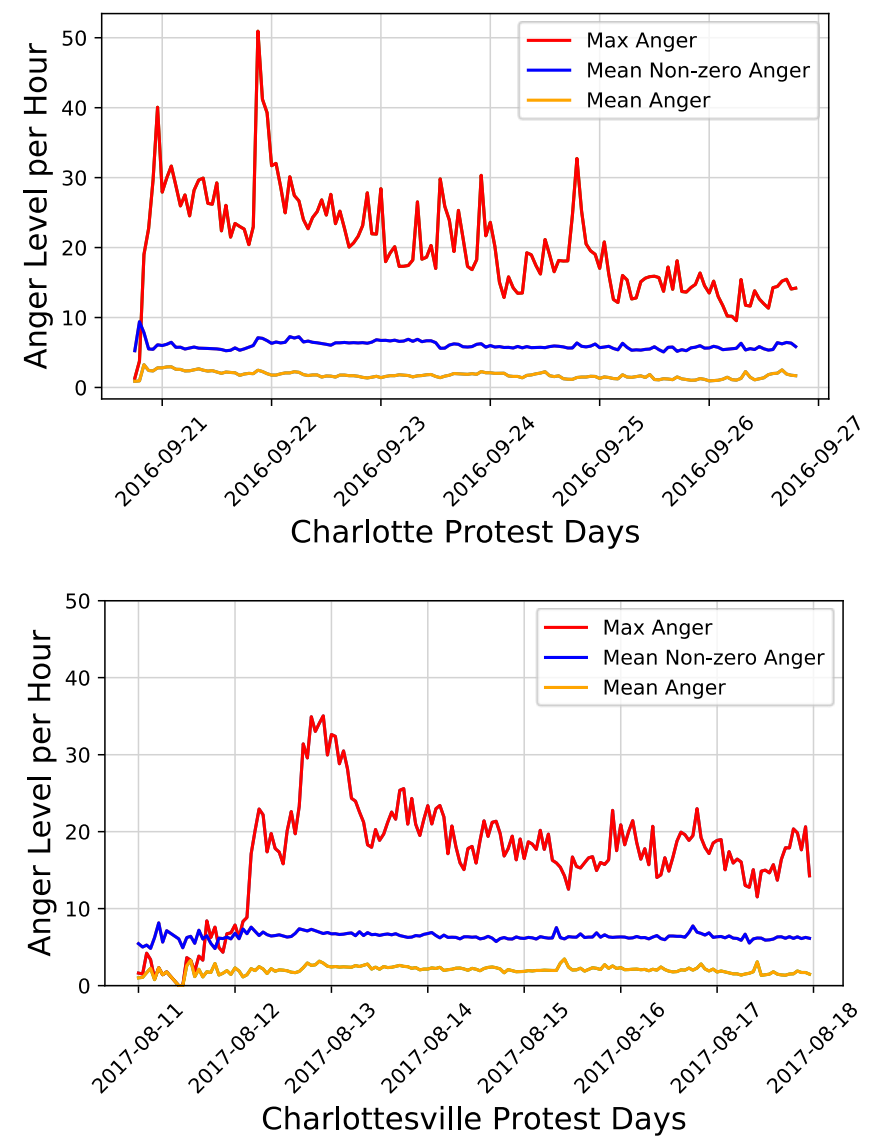

Figure 2. Anger Level Distributions of Tweets in (a) Charlotte Dataset (b) Charlottesville Dataset 
Figure 2(b) illustrates the mean anger per hour of the top ten angriest users (Max Anger), of the 34\% of tweets that showed at least some anger (Mean Non-zero Anger), and of all users (Mean Anger), for the Charlottesville protests. During the third day of protests, the angriest values peak, which likely stemmed from an appearance of the victim's mother in the media. The percentage of users showing some anger in the Charlottesville dataset is $45 \%$. In sum, Charlotte protests tweets are with higher anger levels compared to tweets about Charlottesville protests. However, after early days of both protests, anger dies out. Although these two protests differ in their triggering points, they have similarities in their macro behaviors during the peak protest times.

\subsection{Connection of Network and Anger}

The percentages of influential users in Charlotte and Charlottesville datasets were $11 \%$ and $15 \%$, respectively. The mean anger levels of general public users in Charlotte and Charlottesville datasets were 2.02 and 2.25, respectively. On the other hand, the mean anger levels of influential users in Charlotte and Charlottesville datasets were 1.90 and 2.08, respectively. Our results showed that the angriest users were general public users with the lower number of followers (see Figure 3). In other words, the angriest users had a relatively low impact level in the network: they were rarely retweeted or mentioned by other users. This suggests that directed links might be essential for the spread of influence and anger.
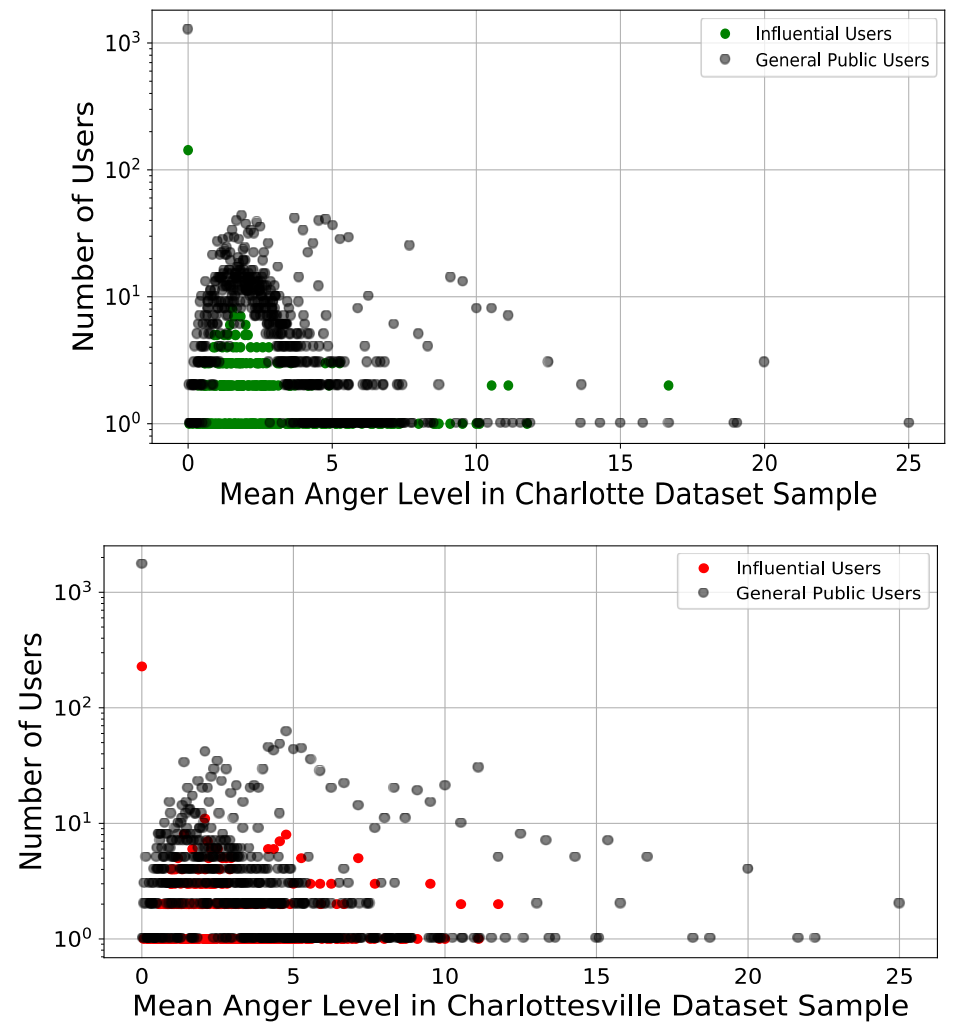

Figure 3. Mean Anger Level Distributions and Users in (a) Charlotte Dataset Sample (b) Charlottesville Dataset Sample

\section{DISCUSSION}

Our results show that despite being two distinct events, the Charlotte and Charlottesville protests follow similar patterns with distributions of anger and mentions/retweets based on their different network structures. Protest-related tweets of influential users (those with more than 10,000 followers) are more often mentioned and retweeted than the tweets of the general public users. Anger levels peak near the beginning of the protest 
and then tend to taper off. The anger levels in Charlotte protests are a bit higher. The angriest users had fewer followers, retweets, and mentions than less angry users. This could be the reason why anger does not persist in the two networks over time. If influential users were more angry, then the anger might have had a chance to persist for a longer time. Different network structures will have different effects on the dynamics of anger. In further work, comparing different network structures and applying these network structures within an agent-based model will help to investigate the effect of different influence algorithms on the spread of anger within online social networks. The law enforcement agencies, governors, and public policy makers can use our analysis to help make decisions about sharing relevant information with the public in a timely manner.

\section{REFERENCES}

Anger, I. \& Kittl, C., 2011. Measuring influence on Twitter. Proceedings of the 11th International Conference on Knowledge Management and Knowledge Technologies. Graz, Austria, p. 31.

Astor, M., Caron, C. \& Victor, D. 2017. A Guide to the Charlottesville Aftermath Video. [Online]. [Accessed 27 March 2019]. Available from: https://www.nytimes.com/2017/08/13/us/charlottesville-virginia-overview.html

Bastian, M., Heymann, S. \& Jacomy, M., 2009. Gephi: an open source software for exploring and manipulating networks. Third International AAAI Conference on Weblogs and Social Media. San Jose, CA, USA, pp. 361-362.

Castells, M., 2015. Networks of outrage and hope: Social movements in the Internet age. John Wiley, Hoboken, NJ, US.

Granovetter, M., 1978. Threshold models of collective behavior. American journal of sociology, 83(6), pp. 1420-1443.

Hu, P. \& Lau, W. C., 2013. A survey and taxonomy of graph sampling. arXiv preprint arXiv:1308.5865.

Jansen, B. J., Zhang, M., Sobel, K. \& Chowdury, A., 2009. Twitter power: Tweets as electronic word of mouth. Journal of the American society for information science and technology, 60(11), pp. $2169-2188$.

Lee, J. C., et al. 2016. Scenes and Voices from the Protests in Charlotte. [Online]. [Accessed 27 March 2019]. Available from: https://www.nytimes.com/interactive/2016/09/22/us/charlotte-police-shooting-protest.html

Mitchell, L., Frank, M.R., Harris, K.D., Dodds, P.S. and Danforth, C.M., 2013. The geography of happiness: Connecting twitter sentiment and expression, demographics, and objective characteristics of place. PloS one, 8(5), p.e64417.

Newman, M. E., 2006. Modularity and community structure in networks. Proceedings of the national academy of sciences, 103(23), pp. 8577-8582.

Pennebaker, J. W., et al., 2015. The development and psychometric properties of LIWC2015.

Taxidou, I. and Fischer, P.M., 2014, April. Online analysis of information diffusion in twitter. In Proceedings of the 23rd International Conference on World Wide Web (pp. 1313-1318). ACM.

Tremayne, M., 2014. Anatomy of protest in the digital era: A network analysis of Twitter and Occupy Wall Street. Social Movement Studies, 13(1), pp.110-126.

Tufekci, Z. \& Wilson, C., 2012. Social media and the decision to participate in political protest: Observations from Tahrir Square. Journal of communication, 62(2), pp. 363-379.

Valenzuela, S. et al., 2014. Facebook, Twitter, and youth engagement: A quasi-experimental study of social media use and protest behavior using propensity score matching. International Journal of Communication, 8, p. 25.

Xie, J. et al., 2011. Social consensus through the influence of committed minorities. Physical Review E, 84(1), p. 011130. 